\title{
Photocatalysis-induced Selective Decoration of Semiconducting Single Walled Carbon Nanotubes: Hole-doping Effect
}

\author{
Satoshi Kubota, ${ }^{\text {a }}$ Daisuke Shimamoto, ${ }^{\mathrm{b}}$ Jin Sung Park, ${ }^{\mathrm{b}}$ Hiromasa Nishikiori, ${ }^{\mathrm{a}}$ Nobuaki Tanaka, ${ }^{\text {a }}$ Yoong \\ Ahm Kim, ${ }^{* a}$ Tsuneo Fujii, ${ }^{\text {a, c }}$ Morinobu Endo ${ }^{\text {a, b }}$ and Milred S. Dresselhaus ${ }^{d}$
}

\author{
${ }_{5}$ Received (in $\left.X X X, X X X\right)$ Xth $X X X X X X X X X$ 200X, Accepted Xth XXXXXXXXX $200 X$ \\ First published on the web $X$ th $X X X X X X X X X 200 X$ \\ DOI: 10.1039/b000000x
}

We examined the time-dependent effect of the titanium oxide photocatalysis on $\mathrm{N}$-methyl-2-pyrrolidone-helped individually ${ }_{10}$ dispersed single walled carbon nanotube (SWNT) suspension. From optical spectroscopic studies, we found a selective decoration of the semiconducting tubes. Such selectivity is attributed to the preferential attack of the photogenerated active species on the hole-doped semiconducting SWNTs.

15 Single walled carbon nanotubes (SWNTs) have attracted considerable attention due to their unique electronic structure as well as their excellent electrical, thermal and mechanical properties. ${ }^{1,2}$ However, such tubes exhibit a strongly bundled structure, and they are insoluble due to their strong 20 hydrophobic nature. Thus, in order to exploit the intrinsically present characteristics of SWNTs, sidewall chemistry has been actively studied to find an efficient way of introducing hydrophilic functional groups on the sidewall and then improving their chemical processibility. Commonly, strong 25 acid treatments have been used to improve the dispersibility of carbon nanotubes. ${ }^{3-6}$ Very recently, titanium oxide photocatalysis has been examined as an alternative tool to modify the sidewall of carbon nanotubes ${ }^{7,8}$ as well as to transform graphene oxide to graphene through a mild 30 process. $^{9-11}$ Even though its effectiveness to modify the sidewall of the tubes was verified, ${ }^{7}$ there is no report on the reaction selectivity of the photogenerated species on metallic and semiconducting SWNTs.

Here, we present a study on the photocatalytic activity of 35 titanium oxide in homogeneously dispersed SWNT suspensions. More specifically, a $N$-methyl-2-pyrrolidone (NMP)-helped individually dispersed SWNT suspension containing titanium oxide thin film was illuminated using ultraviolet light up to 60 days, and then the time-dependent 40 changes in the optical properties of SWNTs were monitored using UV-Vis-NIR absorption spectra. Such photocatalytic treatment selectively depressed the optical activity of semiconducting tubes (due to partially decorated carboxylic

aFaculty of Engineering, Shinshu University, Wakasato, Nagano 3808553,E-mail: yak@endomoribu.shinshu-u.ac.jp; Fax: +81-26-2695208; Tel: +81-26-269-5212;

${ }^{b}$ Institute of Carbon Science \& Technology, Shinshu University, 4-17-1 Wakasato, Nagano 380-8553 (Japan)

${ }^{c}$ Nagano Prefectural Institute of Technology, Shimonogou, Ueda, Nagano 386-1211, Japan

${ }^{d}$ Massachusetts Institute of Technology Cambridge, Massachusetts 02139-4307, USA

$\dagger$ Electronic supplementary information (ESI) available: Figs S1. groups on the sidewall) because the photogenerated active ${ }_{45}$ species (e.g., superoxide-ion and hydroxyl radicals) are expected to have a higher reactivity with the hole-doped SWNTs.

In this study, high-purity Hipco-based SWNTs (ca. 0.5 wt $\%$ metallic impurity) are used without further treatments. ${ }_{50}$ In order to assure homogeneous photocatalytic reactions in the nanotube suspensions, the nanotube suspension was prepared as follows. SWNTs (ca. $2 \mathrm{mg}$ ) were dispersed in NMP (100 $\mathrm{ml}$ ) under strong sonication (UP50H, ca. $600 \mathrm{~W} / \mathrm{cm}^{2}$ ) for $1 \mathrm{hr}$ at $4{ }^{\circ} \mathrm{C}$ and such a nanotube suspension was subjected to 55 ultracentrifugation (Optima Max-XP, Beckman Coulter, $240,000 \mathrm{~g}$ ) in order to remove the bundled tubes. Subsequently, their supernatant $(70 \%)$, rich with isolated nanotubes, was used in our photocatalytic studies.

In addition, titanium oxide film instead of titanium oxide ${ }_{60}$ particles was used as a photo-catalyst in order to avoid direct charge transfer from particles to nanotubes. The titanium oxide film was prepared using the sol-gel method as follows. $15 \mathrm{~mL}$ of tetraisopropyl orthotitanate was slowly added into $15 \mathrm{~mL}$ of ethanol under vigorously stirring in a nitrogen ${ }_{65}$ atmosphere, and then $0.08 \mathrm{~mL}$ of $1.0 \times 10^{-3} \mathrm{~mol} / \mathrm{dm}^{3}$ aqueous hydrochloric solution as a catalyst was slowly added to the solution. After dipping a glass plate in the sol-gel solution, and then thermally treating it at $673 \mathrm{~K}$ for $30 \mathrm{~min}$ in air, we have prepared a titanium oxide film exhibiting crystal 70 structure of anatase (Fig. S1, ESI $\dagger$ ). Finally, the nanotube suspension containing titanium oxide film in a vial was photocatalyzed using ultraviolet light (BL-10, Yazawa cop., $10 \mathrm{~W}$ ) for 10, 30 and 60 days. We have measured the Resonant Raman spectra from this sample using 514.5, 632.8 and 785 $75 \mathrm{~nm}$ laser excitations (Horiba JY T6400) and UV-Vis-NIR absorption spectra (UV-3150, Shimazu) on SWNT suspensions that are photocatalytically treated for different time.

The individually dispersed SWNT suspension is required in 80 order to assure homogenous chemical reactions in SWNTs that are dispersed in NMP by the photocatalysis of titanium oxide. Thus, rather than the commonly used surfactants (e.g., sodium dodecyl benzene sulfonate and sodium dodecyl sulfate), NMP is selected as the dispersing agent, because 85 surfactants are known to adsorb to the sidewall of the tubes and thus might act as a protective layer against photocatalysis. In addition, it is well known that NMP has a high ability to disperse SWNTs up to $0.25 \mathrm{mg} / \mathrm{L} .{ }^{12-14}$ The SWNT suspension that is prepared by the sonication and followed by 
ultracentrifugation was photocatalytically treated for 10,30 and 60 days. The effect of photocatalysis is monitored by observing the UV-Vis-NIR absorption spectra (Fig. 1). The sharp and well-resolved absorption peaks in the pristine 5 sample indicates that the SWNTs were individualized in NMP. The absorption peaks in the range of $400-550 \mathrm{~nm}, 550-900 \mathrm{~nm}$, and 1100-1380 $\mathrm{nm}$ spectral regions can be assigned to the metallic $\mathrm{E}_{11} \quad$ (M1), semiconducting $\mathrm{E}_{22} \quad$ (S2), and semiconducting $\mathrm{E}_{11}(\mathrm{~S} 1)$ transitions, respectively. ${ }^{15}$ Here, $\mathrm{E}_{i i}$ 10 indicates the optical transition energy of an electron-hole pair for the $i$ th valence and $i$ th conduction energy bands. Even though we cannot observed a large change in the M1 and S2 bands in comparison with the pristine sample which is the NMP-helped isolated SWNT suspension before the 15 photocatalytic treatment, the S1 absorption band was drastically changed by the titanium oxide photocatalysis, since the oxidation induced hole-doping effect easily suppresses the electronic transition $\mathrm{S} 1 .{ }^{16}$ We found a time-dependent decrease in the absorption peaks at 1150 and $1294 \mathrm{~nm}$, as well 20 as newly appearing peak at $1172 \mathrm{~nm}$. These results support our conclusion that the S1 band structure in SWNTs was selectively modified by the photocatalytic effect of the

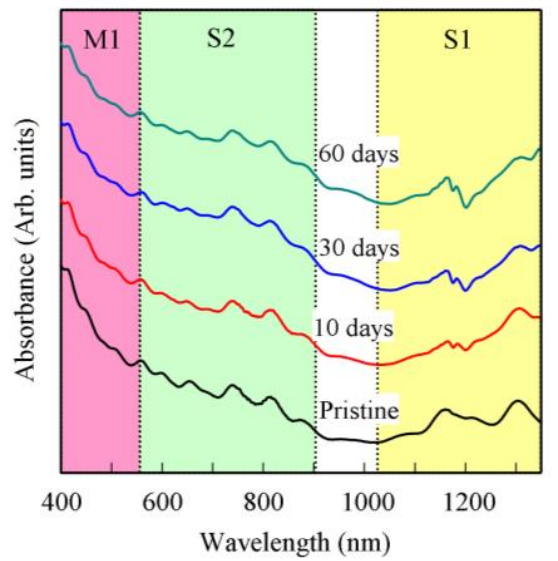

titanium oxide. ${ }^{17}$

Fig. 1 UV-Vis-NIR absorption spectra of the pristine SWNTs and 25 of SWNTs that are photocatalytically treated for 10,30 and 60 days.

In order to study such optical changes in the semiconducting SWNTs in detail, resonance Raman spectroscopy is chosen 30 rather than UV-Vis-NIR absorption spectroscopy because of its high sensitivity to the diameter and the metallicity of the tubes. $^{18,19}$ The SWNT suspension that is photocatalytically treated for 60 days was spin-coated on a glass substrate and then Raman spectra using 514.5, 632.8 and $785 \mathrm{~nm}$ laser 35 excitations were measured (Fig. 2). The assignment of metallic and semiconducting bands was carried out using the Kataura plot. ${ }^{20}$ Figure 2 (a) shows three types of radial breathing modes (RBM) with three different excitation lasers, 785 (red), 632.8 (blue), and $514.5 \mathrm{~nm}$ (black), for the pristine 40 (bold line) and photocatalytically treated samples for 60 days (dotted line). While the pristine sample showed five RBM peaks below $500 \mathrm{~cm}^{-1}$ for the $514.5 \mathrm{~nm}$ laser excitation (Fig. 2 (a)), the photocatalytically treated SWNTs showed severely depressed RBMs at 180 and $210 \mathrm{~cm}^{-1}$ which arise from the
45 semiconducting tubes and three RBMs at 250, 260 and 273 $\mathrm{cm}^{-1}$ coming from the metallic tubes without any distinctive change. Similarly, for the $785 \mathrm{~nm}$ laser excitation, the RBMs in the range of $200-250 \mathrm{~cm}^{-1}$, indicating that the signal from the semiconducting tubes, is depressed via the photocatalytic 50 reactions. However, there is no distinctive change in RBMs at 191 and $220 \mathrm{~cm}^{-1}$ arising from the metallic SWNTs in the Raman spectra taken with a $632.8 \mathrm{~nm}$ laser excitation. It shoud be noted that we did not observe change in the semiconducting tubes with relatively small diameter in the 55 range of $250-300 \mathrm{~cm}^{-1}$ for the 785 and $632.8 \mathrm{~nm}$ laser excitations. This means that the photocatalytic doping effect on the sidewall of SWNT by the titanium oxide occurs selectively for the semiconducting tube with a relatively large diameter over $1 \mathrm{~nm}$ due to the smaller DOS (density of state) 60 at the Fermi level than less than $1 \mathrm{~nm}$ tube and then the quick change of the resonance condition by hole-doping.

Fig. 2 (a) Low-frequency and (b) high-frequency Raman spectra using 514.5, 632.8 and $785 \mathrm{~nm}$ excitation lasers for the pristine
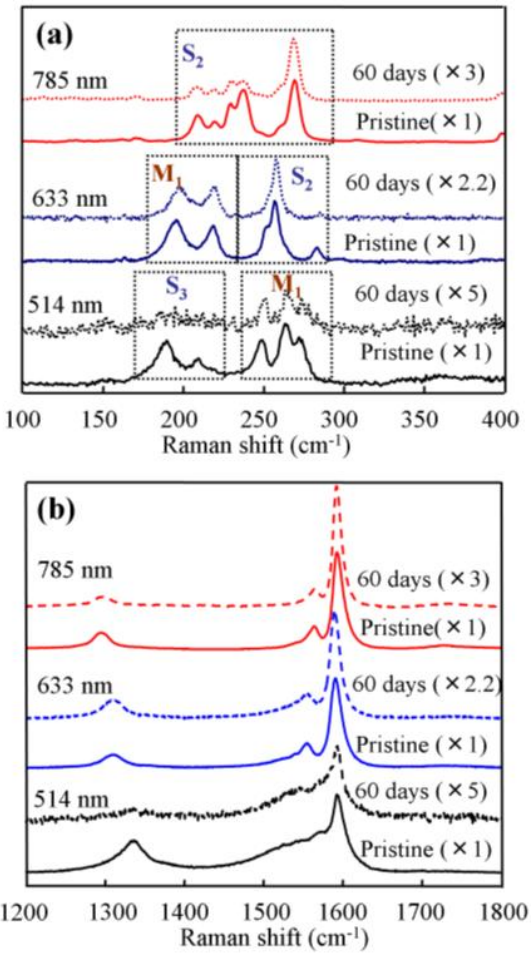

SWNTs and for SWNTs that are photocatalytically treated for 60 65 days. The Raman spectra were normalized to the $\mathrm{G}$ band intensity. $M_{i}$ and $S_{i}$ are metallic and semiconducting interband transitions.

In addition, we are able to notice such selective reactions from high-frequency Raman spectra (Fig. 2 (b)). There is a 70 relatively sharp, strong G-band $\left(\mathrm{E}_{2 \mathrm{~g} 2}\right.$ graphite mode) around $1590 \mathrm{~cm}^{-1}$ and a broad D-band (defect-induced mode) in the range of $1300-1350 \mathrm{~cm}^{-1}$. As shown in the $514.5 \mathrm{~nm}$ Raman spectra of Fig. 2 (b), the photocatalytic treatment gives rise to an intensified Breit-Wigner-Fano (BWF) tail around $1500 \mathrm{~cm}^{-}$ ${ }_{75}{ }^{1}$ which appears only for the metallic tube, supporting the observation that a selective reaction of the photogenerated active species to the semiconducting tubes occurred. 
Conclusively, in the low frequency Raman spectra, the RBM coming from the semiconducting tubes is depressed, while the RBMs from metallic tubes remained the same. From those Raman studies, the depressed Raman lines from 5 semiconducting tubes are the result from the selective reaction of the photogenerated active species on the semiconducting tubes.

It is expected that the hole-doped semiconducting SWNTs have a higher reactivity due to their higher DOS at the Fermi 10 level. Therefore, the reason for such a change in the $\mathrm{S} 1$ band of the UV-Vis-NIR absorption spectra can be explained by hole-doping. In addition, we observed a selective diameter dependent hole-doping effect for semiconducting tubes by the resonance Raman spectroscopy measurements. It should be 15 noted that the absorption spectra are intensive in the small diameter area $(<1 \mathrm{~nm})$ for each $\mathrm{E}_{i i}$ transition due to the lager DOS than more than $1 \mathrm{~nm}$ tubes. Thus, the optical absorption was observed no change for the S2 and S3 spectral regions. As the reason for the selective hole-doping, first, the 20 photocatalysis of titanium oxide generates the electron-hole pair by light irradiation (1). ${ }^{21}$

$\mathrm{TiO}_{2}+\mathrm{h} v \rightarrow \mathrm{h}^{+}+\mathrm{e}^{-}$

${ }_{25}$ Here, $\mathrm{h}$ indicates the Planck constant and $\mathrm{h}^{+}$and $\mathrm{e}^{-}$indicate hole and electron, respectively. The hole-doped SWNTs are formed by the photogenerated hole of the titanium oxide (2), because oxidative treatment is known to induce hole-doping in tubes. $^{22-24}$

30

$\mathrm{SWNT}+\mathrm{h}^{+}\left(\mathrm{TiO}_{2}\right) \rightarrow \mathrm{SWNT}^{+}$

Subsequently, the photo-excited titanium oxide has the ability to reduce $\mathrm{O}_{2}$ and oxidize $\mathrm{H}_{2} \mathrm{O}$ adsorbed on $\mathrm{TiO}_{2}$ or dissolved 35 in NMP. As a result, superoxide-ion $\left(\mathrm{O}_{2}{ }^{-}\right)$and hydroxyl radical $(\cdot \mathrm{OH})$ are generated $(3)$ :

$\mathrm{O}_{2}+\mathrm{e}^{-}\left(\mathrm{TiO}_{2}\right) \rightarrow \mathrm{O}_{2}^{-}$

$\mathrm{H}_{2} \mathrm{O}+\mathrm{h}^{+}\left(\mathrm{TiO}_{2}\right) \rightarrow \cdot \mathrm{OH}+\mathrm{H}^{+}$

Finally, photogenerated active species such as $\mathrm{O}_{2}{ }^{-}$and $\cdot \mathrm{OH}$ chemically attack the hole-doped semiconducting SWNTs. Eventually, oxygen-containing groups such as the carboxyl group are sequentially decorated on the sidewall of the 45 semiconducting SWNTs (4), thereby resulting in the breakdown of van Hove singularities (and a loss of Raman resonance).

$$
\mathrm{SWNT}^{+} \text {(semiconducting) }+\mathrm{O}_{2}^{-} \text {and } \stackrel{\mathrm{OH}}{\rightarrow} \rightarrow
$$$$
50
$$

In summary, using optical spectroscopies, we demonstrated that the titanium oxide photocatalysis showed a unique chemistry to introduce oxygen functional groups 55 preferentially on the sidewall of the semiconducting tubes with large diameter $(>1 \mathrm{~nm})$ in an NMP-helped individually dispersed SWNT suspension. The drastic change in the S1 band of the absorption spectra as well as the depressed intenisty for the Raman lines arising from the semiconducting ${ }_{60}$ tubes supports the claim that the photogenerated species (e.g., superoxide-ion and hydroxyl radicals) selectively attach to the hole-doped semiconducting SWNTs. We envisage that our study will be helpful for manipulating the diameter and the metallicity of SWNTs in a more controlled manner.

${ }_{65}$ We acknowledge the support from the Regional Innovation Cluster Program of Nagano and a Grant-in-Aid (Nos. 19002007, 20510096 and 22915010) from the Ministry of Education, Culture, Sports, Science and Technology of Japan. MSD acknowledges support from US NSF Grant DMR-077004197.

\section{Notes and references}

1. S. J. Tans, M. H. Devoret, H. Dai, A. Thess, R. E. Smalley, L. J. Geerligs and C. Dekker, Nature, 1997, 386, 474.

2 R. Saito, G. Dresselhaus and M. S. Dresselhaus, Phys. Rev. B, 2000 $75 \quad 61,2981$.

3 D. B. Mawhinney, V. Naumenko, A. Kuzenetsova, J. T. J. Yates, J. Liu and R. E. Smalley, J. Am. Soc., 2000, 122, 2382

4 S. Nagasawa, M. Yudasaka, K. Hirahara, T. Ichihashi and S. Iijima, Chem. Phys. Lett., 2000, 328, 374

805 H. Hu, B. Zhao, M. E. Itkis, and R. C. Haddon, J. Phys. Chem. B, 2003, 107, 13838

6 J. Zhang, H. Zou, Q. Qing, Y, Yang, Q. Li, Z. Liu, X. Guo and Z. Du, J. Phys. Chem. B, 2003, 107, 3712.

7 S. H. Lee, Y. C. Jung, Y. A. Kim, H. Muramatsu, K. Teshima, S. 85 Oishi and M. Endo, Nanotechnology, 2009, 20, 105708.

8 S. H. Lee, K. Teshima, I. Y. Jang, K. Yubuta, Y. J. Kim, Y. A. Kim, T. Shishido, M. Endo and S. Oishi, Chem. Commun., 2010, 46, 2295.

9 X. Zhang, H. Kono, Z. Liu and A. Fujishima, Chem. Commun., 90 2007, 4949.

10 B. Li, X. Zhang, X. Li, L. Wang, R. Han, B. Liu, W. Zheng, X. Li and Y. Li, Chem. Commun., 2010, 46, 3499.

11 G. Williams, B. Seger and P. V. Kamat, ACS Nano, 2008, 7, 1487.

12 K. D. Ausman, R. Piner, O. Lourie, R. S. Ruoff and M. Korobov, $J$. 95 Phys. Chem. B, 2000, 104, 8911.

13 S. Giordani, S. D. Bergin, V. Nicolosi, S. Lebedkin, M. M. Kappes, W. J. Blau and J. N. Coleman, J. Phys. Chem. B, 2006, 110, 15708.

14 J. L. Bahr, E. T. Mickelson, M. J. Bronikowski, R. E. Smalley and J. M. Tour, Chem. Comm., 2001, 193.

10015 M. J. O'Connell, S. M. Bachilo, C. B. Huffman, V. C. Moore, M. S Strano, E. H. Haroz, K. L.Rialon, J. Boul, W. H. Noon, C. Kittrell, J. Ma, R. H. Hauge, R. B. Weisman and R.E. Smalley, Science, 2002, 297, 593.

16 M. E. Itkis, S. Niyogi, M. E. Meng, M. A. Hamon, H. Hu, and R. C. 105 Haddon, Nano Lett., 2002, 2, 155.

17 Y. Miyata, Y. Maniwa and H. Kataura, J. Phys. Chem. B, 2006, 110, 25.

18 M. S. Dresselhaus and P. C. Eklund, Advances in Physics, 2000, 49 , 705 .

11019 A. Jorio, R. Saito, J. H. Hafner, C. M. Lieber, M. Hunter, T. McClure, G. Dresselhaus and M. S. Dresselhaus, Phys. Rev. Lett., 2001, 86, 1118.

20 H. Kataura, Y. Kumazawa, Y. Maniwa, I. Umezu, S. Suzuki, Y. Ohtsuka and Y. Achiba, Synthetic Metals, 1999, 103, 3555.

11521 Z. Yu and S. S. C. Chuang, App.Cata. B Env., 2008, 83, 277.

22 J. Liu, A. G. Rinzler, H. Dai, J. H. Hafner, R. K. Bradley, P. J. L. Boul, A. T. Iverson, K. Shelimov, C. B. Huffman, F. RodriguezMacias, Y.-S. Shon, T. R. Lee, D. T. Colbert and R.E. Smalley, Science, 1998, 280, 1253.

12023 S. Ramesh, L. M. Ericson, V. A. Davis, R. K. Saini, C. Kittrell, M. Pasquali, E. Billups, W. W. Adams, R. H. Hauge and R. E. Smalley, J. Phys. Chem. B, 2004, 108, 8794 .

24 W. Zhou, J. Vavro, N.M. Nemes, J.E. Fischer, F. Borondics, K. Kamaras and D. B. Tanner, Phys. Rev. B, 2005, 71, 205423. 